\title{
Provider Bias in Prescribing Opioid Analgesics: An Analysis of Emergency Department Electronic Medical Records
}

Brian Aronson ( $\sim$ bdaronson@gmail.com )

Indiana University Bloomington https://orcid.org/0000-0001-5304-1971

Lisa A Keister

Duke University

James Moody

Duke University

Research article

Keywords: Opioids, Prescription bias, Emergency departments, Electronic medical records, Undertreatment, Crowding, Inequality

Posted Date: April 13th, 2020

DOl: https://doi.org/10.21203/rs.3.rs-20738/v1

License: (9) This work is licensed under a Creative Commons Attribution 4.0 International License. Read Full License

Version of Record: A version of this preprint was published at BMC Public Health on August 6th, 2021. See the published version at https://doi.org/10.1186/s12889-021-11551-9. 


\section{Abstract}

Background: Physicians do not prescribe opioid analgesics for pain treatment equally across groups, and such disparities may pose significant public health concerns. While research suggests that institutional constraints and cultural stereotypes influence doctors' treatment of pain, prior quantitative evidence is mixed. The objective of this secondary analysis is therefore to clarify which institutional constraints and patient demographics truly bias provider prescribing of opioid analgesics.

Methods: We used electronic medical record data from an emergency department of a large U.S hospital during years 2008-2014. We ran multi-level logistic regression models to estimate factors associated with providing an opioid prescription during a given visit while controlling for ICD-9 diagnosis and betweenpatient heterogeneity.

Results: A total of 180,829 patient visits among 65,513 unique patients were recorded during the period of analysis. Overall, providers were significantly less likely to prescribe opioids to the same individual patient when the visit occurred during higher rates of emergency department crowding, earlier times of day, earlier times of the week, later years, and when the patient had received fewer previous opioid prescriptions. Across all patients, providers were significantly more likely to prescribe opioids to patients who were middle-aged, white, and married. We found no bias towards women and no interaction effects between race and crowding or between race and gender.

Conclusions: Providers tend to undertreat pain during constrained diagnostic situations and undertreat pain for patients from high-risk and marginalized demographic groups. Harm from previous treatment mistakes are likely to accumulate through informing future treatment decisions.

\section{Background}

Physicians do not prescribe opioid analgesics for pain treatment equally across groups. Latino and African Americans, for example, are significantly less likely than whites to receive opioid analgesics following a major surgical procedure (1-4), and opioid prescriptions rates for women and senior citizens appear low relative to their reports of chronic pain (4-6). Such disparities in pain treatment may pose significant public health concerns. Many recognize pain as the "fifth vital sign" of health $(7,8)$; undertreatment of pain is associated with a variety of disadvantages such as diminished quality of life, physical functioning, mental acuity, sexual functioning, and sleep. Undertreated pain is also associated with increased (and costly) returns to the hospital $(9,10)$. Therefore, it is critical to identify factors that might bias doctors' treatment of pain.

Undertreatment for pain results from many factors that operate in combination including pain's ambiguous diagnostic criteria, cultural stereotypes around particular groups' experience of pain, and institutional constraints that affect doctors' ability to diagnose pain. Because pain often lacks clear physical markers, doctors are forced to elicit information directly from patients to determine whether opioid analgesic treatment is necessary (11-13). However, subjective assessments of patients' pain needs 
are highly subject to implicit bias (14). For example, doctors often mistakenly assume that African Americans have more pain tolerance and have more illicit motivations for seeking pain treatment than do whites (15-19). Long-standing stereotypes about women's lack of pain tolerance appear to bias doctors to both over-treat and undertreat women's pain $(4,20)$. Preferences for patients who are culturally similar to doctors may lead doctors to reduce attention to the suffering of patients from lower socio-economic groups $(11,16,21,22)$ thereby limiting doctors' ability to make informed diagnoses. Institutional constraints also play a role in doctors' treatment decisions. Some evidence suggests that doctors are more likely to over-treat pain during periods of increased time-pressure, such as when it is late at night or when emergency departments are crowded $(15,23)$. Such time constraints may intensify doctors' implicit biases by increasing their likelihood to rely on implicit assumptions about patient diagnostic categories to inform treatment $(13,24)$.

Although there are many studies about how institutional constraints and cultural stereotypes influence doctors' treatment of pain, quantitative evidence is mixed. Recent research suggests that doctors are biased towards over-treating patients during periods of time constraint and hospital crowding $(15,23)$, but such claims seem to contradict a small body of evidence that doctors are averse to prescribing opiates when under time-constraint $(4,25)$. Gendered biases in pain treatment have been documented in a fairly large body of ethnographic research $(4,20)$, but these biases are not well-supported by quantitative evidence $(3,26,27)$. In fact, even though race may be the best documented source of provider bias in opioid pain treatment, prior studies have only examined these biases in reference to Latino and African American patients. There have been no substantial attempts to explain how other racial backgrounds, such as Asian heritage, is associated with pain treatment.

Sociological research suggests that some discrepant findings may be due to overlooked mediators and moderators of other cultural and institutional constraints $(14,15,20,28)$. For example, high frequency of opioid treatment of pain for patients late at night may reflect selection biases in patients' medical histories rather than doctors' time pressures. Many demographic correlates with pain treatment might be induced by related factors, such as such as patient race, gender, age, marital status, or medical history. Ignoring how multiple factors simultaneously contribute to bias in pain treatment likely subjects much prior research to omitted variable bias.

To understand how institutional constraints and patient demographics influence pain treatment, this paper examines a large longitudinal dataset of electronic medical records gathered at an emergency department of a large private hospital in the United States. We study how opioid treatment for pain is simultaneously influenced by multiple factors and how this may bias pain treatment. Specifically, we examine how pain treatment is influenced by the intersection of emergency department crowding and race, the time patients visited the emergency department, patients' medical histories, patients' diagnoses, age, sex, marital status, and year of analysis. This is one of the largest and most comprehensive longitudinal study of demographic and institutional predicates of opioid pain treatment in the literature, and it is one of only a few studies that examine multiple factors associated with pain treatment in 
tandem and over time. Our findings inform dominant hypotheses about provider bias, clarify prior mixed evidence, and provide evidence for under-explored avenues of bias in pain treatment.

\section{Methods}

We assess provider biases in pain treatment with data from an electronic medical record (EMR) system of a large university hospital in the United States. We focus our analysis on EMR data collected from 20082014 at the hospital's emergency department (ED) ( $n=180,829$ patient visits; 65,513 unique individuals). Consistent with prior work on doctor biases in pain treatment, we focus on EDs because the majority of patients who visit EDs (prior estimates as high as $70 \%(29,30)$ ) do so for pain treatment. EDs are also heavily subject to crowding and often serve patients that come from a wide array of socioeconomic backgrounds - both factors that are critical to our analysis.

\section{Statistical Analyses}

To determine factors associated with provider bias in opioid pain treatment, we conduct a series of three generalized linear mixed models with a logit link function that predict whether a patient was prescribed opioids during a given ED visit based on a variety of contextual and demographic variables. Our models are ordered by complexity. Our first model includes within-patient random intercepts to account for repeated observations among individual patients. Our second model includes both within-person random intercepts and fixed-effect controls for International Classification of Diseases(31) (ICD-9) diagnoses to account for differences in patients' medical needs. Controlling for patient diagnoses should prevent spurious associations between opioid treatment and patient characteristics through the association of particular medical conditions with particular demographic backgrounds. Our final model controls for within-patient fixed effects and ICD-9 diagnoses. Including within-patient fixed effects allows us to closely track how changes in the medical environmental affect providers' likelihood to prescribed opioid analgesics to individual patients; however, this prevents us from estimating how time-invariant factors (e.g. patient race) are associated with opioid treatment.

\section{Measures}

The dependent variable in all models is whether individuals were prescribed any opioids during the patient visit. This is a binary measurement that tracks whether a patient received at least one of the following generic opioid prescriptions (listed in order of frequency): oxycodone, morphine, hydromorphone, fentanyl, hydrocodone, codeine, methadone, tapentadol, or meperidine.

We use five variables to measure context. First, we measure ED crowding, our most central contextual measure, as the number of patients who visited in the last four hours. This variable accounts for the degree to which providers are under time-pressure. We chose four hours as our cutoff in order to leverage available data and account for the fact that many EDs set four-hours as their target maximum wait time. (32) Second, we test whether ED crowding is more strongly associated with prescribing opioids to patients based on patient race by including an interaction between $E D$ crowding and respondent race (ED 
crowding $x$ black). Third, we include an indicator that the patient arrived between 12am-6am to control for lower patient counts after midnight. Fourth, we include an indicator that the ED visit occurred on a weekend to control for lower patient numbers during the weekend, and finally, we include an indicator for year of visit (reference $=2008$ ) to account for a cautioning effect of the opioid crisis on prescribing opioids.

We also control demographic factors that are known to be associated with opioid prescriptions. We include measures of patient age coded as age 30-60 and age-60+ (reference $=$ Age $<30$ ) to test whether providers are more hesitant to prescribe opioid analgesics to young and older citizens. We measure race with four dichotomous variables: black, Latino, Asian and other to test for racial biases against prescribing opioid towards nonwhites. We include a term for female and we include four race-female interaction terms to test whether women or women of minority races have distinct disadvantages when seeking pain treatment. We include variables for marital status (married, divorced, widowed, and separated) to test whether doctors prescribe opioid analgesics more often to individuals who have familial ties than to individuals who are single. Finally, we account for patient medical histories by modeling the number of times patients had received any opioid prescriptions during prior ED visits (previously prescribed number). Because our coverage of patients' medical histories increases over time, we also include year interactions with patient prescription counts and exclude the first year of our available data (2007) from analysis.

\section{Results}

Table 1 presents descriptive statistics of all variables. Consistent with prior estimates of opioid prescriptions at EDs, one-third of patient visits to the ED resulted in an opioid prescription. The majority of patient visits were by individuals who were female (0.57), black (0.60) and single (0.53). These numbers partially reflect a greater propensity for female and black individuals to visit EDs than males or whites and partially reflect the demographics of individuals in the county (excluded for confidentiality).

Figure 1 illustrates patterns of ED visits over time. There are relatively minimal annual and monthly trends in ED crowding, with ED crowding appearing smallest towards the end of the year and the beginning of the month. However, the ED was noticeably less crowded towards the end of the week and during late hours of the day. These results suggest that conditioning for the time of a patients' visit may be necessary for determining the true association between ED crowding and opioid prescribing.

Table 2 provides three mixed models for opioid prescribing during a given ED visit, with Model 2 controlling for ICD-9 diagnosis and Model 3 controlling for all unobserved heterogeneity between patients. Parameter estimates are reported in average marginal effects, with a 0.01 unit increase in a given parameter representing a one-percentage point increase in the probability that the provider will prescribe opioids. Parameter estimates in the original models are reported in log odds, include polynomial terms and interaction effects for previously prescribed number and year, and are available in Additional File 1. 
Parameter estimates for contextual variables are similar across models. ED crowding was associated with a reduced probability for providers to prescribe opioid ( $b=-0.002$ in Model 3), suggesting that providers are less likely to prescribe opioids during crowded hours. The coefficient for ED crowding implies that patients who visited the ED during mean rates of crowding (13.92 patients) had a $2.78 \%$ reduced probability to receive an opioid prescription than patients who visited the ED while it was empty. This negative association holds even when we attempted to include an interaction effect between ED crowding and time of day. In contrast, there is no significant association for the interaction between ED crowding and black patients across models ( $b=0.000$ in Model 3$)$, indicating no significant difference in how ED crowding affected provider prescribing towards blacks and whites. The positive). The parameter estimates for 12am-6am ( $b=0.020$ in Model 3 ) indicate and weekend ( $b=0.021$ in Model 3) are positive, indicating that providers were more likely to prescribe opioids during the evening than during the day. The positive association between weekend and opioid prescribing ( $b=0.021$ in Model 3 ) means that providers were more likely to prescribe opioids towards the end of the week. In addition, the coefficients for year are negative ( $b=-0.038$ in Model 3$)$, implying that providers have become less likely to prescribe opioids than in previous years. Moreover, the models imply that providers were substantially more likely to prescribe opioids to patients that had been previously prescribed opioids at the ED $(b=0.027$ in Model 3).

Parameter estimates for demographic variables are unavailable for Model 3 due to their time-invariance but are mostly consistent between Model 1 and Model 2. Age has a curvilinear relationship with opioid prescribing, with individuals' probability to receive opioids increasing during ages 30-60 $(b=0.023$ in Model 2) and decreasing following age 60 ( $b=-0.047$ in Model 2). Patients that are married $(b=0.015)$ and divorced $(b=0.015)$ have an increased probability to be prescribed opioids. On the other hand, there is no significant association for opioid prescribing among females or any female-race interaction variables. Without controlling for ICD-9 diagnosis (as illustrated in Model 1), providers have a lower probability to prescribe opioids to patients that are black $(b=-0.017)$ and Asian $(b=-0.068)$, and a higher probability to prescribe opioids to individuals who are Latino $(b=0.015)$. However, controlling for ICD-9 diagnosis in Model 2 resulted in negative parameter estimates for all non-white race categories $(b=$ -0.005 for blacks; $b=-0.009$ for Latinos; $b=-0.055$ for Asians; $b=-0.003$ for individuals of other race) and resulted in statically significant effects only for individuals who are Asian. Together, these changes indicate a negative association between non-white race and receiving opioid analgesics once we control for racial differences in health conditions.

\section{Discussion}

Prior research has identified multiple factors that can bias provider prescribing of opioids; however, evidence is often mixed. We theorized that previous discrepant findings might result from overlooked mediators and moderators of prescribing behavior. Therefore, we modeled how multiple institutional and cultural constraints work in tandem to influence provider prescription of opioids with data from an emergency department's electronic medical records. Overall, our results may provide explanations to previous inconsistencies, and suggest new factors that might influence opioid prescribing. 
Most centrally, we found that doctors are less likely to prescribe opioids during ED crowding, and we found no race interaction with ED crowding, implying that ED crowding reduces provider opioid prescription rates towards all races. We attribute this finding to risk-aversion: providers are aware that opioid analgesics have severe negative side effects, and they err on the side of caution when their ability to evaluate patient needs is constrained $(4,25)$. Our risk-aversion hypothesis is consistent with our findings that providers are less likely to prescribe opioids to greater risk demographics (young and old patients), lower-information patients (those with fewer previous opioid prescriptions), and during periods of time where the harms of opioid analgesics are more salient (later years of analysis). Conflicting findings likely arise from differences in study samples. For example, Neprash and Barnett (23) focused their analysis on a sample of pre-scheduled primary care office visits of patients that had a history with a given doctor. Compared to providers in EDs, providers in primary care offices have a greater amount of information about their patients and a much stronger motivation towards maintaining those relationships. Perhaps in such contexts, providers' aversion to displeasing their clientele surpasses their aversion to over-prescribing opioid analgesics. Likewise, Lara-Millán's (15) ethnographic study of ED crowding and opioid prescribing for black patients was drawn from a hospital that appeared to be underresourced. Perhaps underfunding further influences providers away from prioritizing quality care.

This study also produced four novel findings. First, even net of diagnoses and between-patient heterogeneity, we found a strong preference for doctors to prescribe opioids to patients who had received opioids at the ED before. This suggests that providers rely heavily on past treatment information to inform future treatment decisions, and importantly, implies that the harm of a single treatment mistake could be compounded with its effect on the likelihood of making future mistakes. Second, we found strong weekly and daily patterns of ED crowding. Even though EDs are designed for emergencies, we believe these patterns reflect periods where patients are more likely to have an injury and reflect patients' preferences to visit the ED during non-recreational periods. Third, we found that doctors were more likely to prescribe opioids during late hours of the day and later days of the week. These trends likely represent a selection effect: patients who visit the ED during inconvenient periods of time likely have true physical emergencies. Even though we controlled for patient ICD-9 diagnoses, these classifications likely fail to wholly capture patients' needs for analgesic treatment. Finally, we found that providers are less likely to prescribe opioid analgesics to all racial minorities, especially towards Asians. We have little ability to distinguish whether these treatment decisions were influenced by provider stereotypes or by norms of stoicism among minority patients. In either case, these disparities likely imply that racial minorities are undertreated for pain.

Most other findings were consistent with the literature. Individuals with valued familial ties, such as those who are married or divorced, were significantly more likely to be prescribed opioid analgesics. Higher-risk age groups, such as children and seniors, were significantly less likely to receive opioids. Patients who visited the ED in later years were less likely to receive opioid analgesics, indicating a cautioning effect of the opioid crisis. In addition, we found no evidence for female or female-race interaction effects on opioid analgesic prescribing. This null finding does not necessarily contradict studies which find a female effect on opioid prescribing; however, it suggests that gender biases in opioid treatment may only occur under 
particular diagnostic contexts. Future research should further attempt to identify what types of contexts produce stronger gender biases.

This study had several limitations. First, the study only examined prescribing habits during the years 2008-2014; factors associated with prescribing bias may have changed since this period. However, many sources of provider bias that we had observed have been noted in the literature for decades. Likewise, we conducted supplemental analyses of prescriber biases with data only from 2008 and data only from 2014 and we found substantively identical results to those reported in the manuscript at both time points. Put together, this evidence suggests that the factors we identified to be associated with prescriber bias during 2008-2014 are likely to be highly stable over time and still present today. Second, the study only examined prescribing habits in one hospital ED. The ED of our analysis is unlikely to be representative of all other EDs in the United States; our sample is better funded than most EDs and the demographic composition of our ED's patients over-represents women, African Americans, and unmarried individuals. Third, the analysis was conducted on secondary data rather than on experimental results, thereby limiting the analysis' ability to differentiate factors that may cause provider bias from factors that are simply correlated with provider bias. Fourth, our measurement of ED crowding is limited in that it does not directly measure the number of patients in an ED waiting room or the amount of time that each patient was seen by a given provider. We assume that EDs became was more crowded when increasing numbers of patients visited the ED net of time of day and week; however, daily variations in the time that some patients took to treat and the number of providers available in the ED almost certainly weakened the accuracy of our measurement of ED crowding. Lastly, much of our discussion assumes that factors which reduce providers' likelihood to prescribe opioids leads to undertreatment; however, data limitations (and the inherent ambiguity of opioid diagnosis) prevent us from determining whether patients in these conditions were truly undertreated. Factors that we found to have a downward association with opioid pain treatment may have actually served to prevent overtreatment.

\section{Conclusions}

We found that within-patient differences in the receipt of an opioid prescription during a visit to the ED was negatively associated with visiting the ED at higher rates of crowding, earlier times of day, earlier times of the week, at later years, and with having received few opioid prescriptions during previous ED visits. Across patients, we found that providers were more likely to prescribe opioids to patients who were middle-aged, white, and married. These results reaffirm previous findings that institutional constraints and racial demographics can significantly bias how providers prescribe opioid analgesics and suggest new paths that may bias providers in analgesic treatment. Given the current state of the opioid epidemic in America, our findings that providers tend to err towards undertreatment for pain during constrained diagnostic situations may seem encouraging. However, it is critical that we do not forget the costs of undertreatment as well. To better-emphasize the costs of undertreatment, future research should attempt to estimate the direct costs of provider biases in opioid analgesic prescribing on patients' long-term health. It is essential that the field continues to work towards identifying institutional policies that can lead to consistent and appropriate treatment for pain. 


\section{Abbreviations}

EMR: Electronic Medical Records

ED: Emergency Department

ICD-9: International Classification of Diseases

\section{Declarations}

\section{Availability of data and materials}

The dataset generated and analyzed during the current study is not publicly available to protect the privacy of participants but it is available from the corresponding author on reasonable request.

\section{Acknowledgements}

Not applicable

\section{Ethics Declarations}

\section{Ethics approval and consent to participate}

This study received Institutional Review Board (IRB) approval. (DUHS Institutional Review Board approval on January 4, 2019 for protocol Pro00084889.) This study was deemed minimal risk by the IRB and the request of Health Insurance Portability and Accountability Act (HIPAA) Waiver of Authorization for recruitment was reviewed and approved.

\section{Consent for publication}

Not applicable.

\section{Competing interests}

The authors declare that they have no competing interests.

\section{Funding}

No funding was obtained for this study

\section{Authors' contributions}

All authors made significant contributions to this project. BA, LK, and JM contributed to the study conception. JM assisted in data acquisition. BA ran statistical analysis. LK and JM consecutively commented on data analysis. BA led drafting of manuscript with assistance and revisions from LK. All authors have read and approved the final manuscript. 


\section{References}

1. Tamayo-Sarver JH, Hinze SW, Cydulka RK, Baker DW. Racial and ethnic disparities in emergency department analgesic prescription. Am J Public Health. 2003;93(12):2067-73.

2. Goyal MK, Kuppermann N, Cleary SD, Teach SJ, Chamberlain JM. Racial disparities in pain management of children with appendicitis in emergency departments. JAMA Pediatr. 2015;169(11):996-1002.

3. Green CR, Anderson KO, Baker TA, Campbell LC, Decker S, Fillingim RB, et al. The unequal burden of pain: confronting racial and ethnic disparities in pain. Pain Med. 2003;4(3):277-94.

4. Pines JM, Hollander JE. Emergency department crowding is associated with poor care for patients with severe pain. Annals of Emergency Medicine. 2008;51(1):1-5.

5. Werner A, Isaksen LW, Malterud K. 'I am not the kind of woman who complains of everything': Illness stories on self and shame in women with chronic pain. Social Science \& Medicine. 2004;59(5):103545.

6. Landi F, Onder G, Cesari M, Gambassi G, Steel K, Russo A, et al. Pain management in frail, community-living elderly patients. Arch Intern Med. 2001;161(22):2721-4.

7. Merboth MK, Barnason S. Managing pain: the fifth vital sign. Nurs Clin North Am. 2000;35(2):375-83.

8. Volkow ND, Frieden TR, Hyde PS, Cha SS. Medication-assisted therapies-tackling the opioidoverdose epidemic. New England Journal of Medicine. 2014;370(22):2063-6.

9. Sinatra R. Causes and consequences of inadequate management of acute pain. Pain Med. 2010;11(12):1859-71.

10. Coley KC, Williams BA, DaPos SV, Chen C, Smith RB. Retrospective evaluation of unanticipated admissions and readmissions after same day surgery and associated costs. J Clin Anesth. 2002;14(5):349-53.

11. Hirsh AT, Hollingshead NA, Ashburn-Nardo L, Kroenke K. The interaction of patient race, provider bias, and clinical ambiguity on pain management decisions. J Pain. 2015;16(6):558-68.

12. Jacobsson K. Categories by heart: Shortcut reasoning in a cardiology clinic. Professions and Professionalism. 2014;4(3).

13. Diamond-Brown L. The doctor-patient relationship as a toolkit for uncertain clinical decisions. Social Science \& Medicine. 2016;159:108-15.

14. Spencer KL, Grace M. Social foundations of health care inequality and treatment bias. Annual Review of Sociology. 2016;42:101-20.

15. Lara-Millán A. Public emergency room overcrowding in the era of mass imprisonment. American Sociological Review. 2014;79(5):866-87.

16. Sabin JA, Greenwald AG. The influence of implicit bias on treatment recommendations for 4 common pediatric conditions: pain, urinary tract infection, attention deficit hyperactivity disorder, and 
asthma. Am J Public Health. 2012;102(5):988-95.

17. Burgess DJ, van Ryn M, Crowley-Matoka M, Malat J. Understanding the provider contribution to race/ethnicity disparities in pain treatment: insights from dual process models of stereotyping. Pain Med. 2006;7(2):119-34.

18. Hoffman KM, Trawalter S, Axt JR, Oliver MN. Racial bias in pain assessment and treatment recommendations, and false beliefs about biological differences between blacks and whites. Proceedings of the National Academy of Sciences. 2016;113(16):4296-301.

19. Hinze SW, Webster NJ, Chirayath HT, Tamayo-Sarver JH. Hurt running from police? No chance of (pain) relief: The social construction of deserving patients in emergency departments. Social Sources of Disparities in Health and Health Care and Linkages to Policy, Population Concerns and Providers of Care: Emerald Group Publishing Limited; 2009. p. 235-61.

20. Pryma J. “Even my sister says I'm acting like a crazy to get a check”: Race, gender, and moral boundary-work in women's claims of disabling chronic pain. Social Science \& Medicine. 2017;181:6673.

21. Shim JK. Cultural health capital: A theoretical approach to understanding health care interactions and the dynamics of unequal treatment. J Health Soc Behav. 2010;51(1):1-15.

22. Gengler AM. “I want you to save my kid!” illness management strategies, access, and inequality at an elite university research hospital. Journal of Health and Social Behavior. 2014;55(3):342-59.

23. Neprash HT, Barnett ML. Association of primary care clinic appointment time with opioid prescribing. JAMA Netw Open. 2019;2(8):e1910373.

24. Stepanikova I. Racial-ethnic biases, time pressure, and medical decisions. J Health Soc Behav. 2012;53(3):329-43.

25. Hwang U, Richardson LD, Sonuyi TO, Morrison RS. The effect of emergency department crowding on the management of pain in older adults with hip fracture. J Am Geriatr Soc. 2006;54(2):270-5.

26. Weisse CS, Sorum PC, Dominguez RE. The influence of gender and race on physicians' pain management decisions. J Pain. 2003;4(9):505-10.

27. Weisse CS, Sorum PC, Sanders KN, Syat BL. Do gender and race affect decisions about pain management? Journal of General Internal Medicine. 2001;16(4):211-7.

28. Pescosolido BA, Martin JK, McLeod JD, Rogers A. Handbook of the Sociology of Health, IIIness, and Healing: A Blueprint for the 21st Century: Springer Science \& Business Media; 2010.

29. Duignan M, Dunn V. Barriers to pain management in emergency departments. Emerg Nurse. 2008;15(9):30-4.

30. Tcherny-Lessenot S, Karwowski-Soulie F, Lamarche-Vadel A, Ginsburg C, Brunet F, Vidal-Trecan G. Management and relief of pain in an emergency department from the adult patients' perspective. $J$ Pain Symptom Manage. 2003;25(6):539-46.

31. International Classification of Diseases, 9th Revision, Clinical Modification. Geneva, Switzerland: World Health Organization; 1980. 
32. Higginson I. Emergency department crowding. Emerg Med J. 2012;29(6):437-43.

\section{Tables}

Table 1 Descriptive Statistics

\begin{tabular}{|c|c|c|c|c|}
\hline & Mean & SD & Min & Max \\
\hline \multicolumn{5}{|l|}{ Contextual Variables } \\
\hline Prescribed opioid ${ }^{\mathrm{a}}$ & 0.33 & 0.47 & 0.00 & 1.00 \\
\hline ED crowding ${ }^{b}$ & 13.99 & 6.30 & 0.00 & 52.00 \\
\hline $12 \mathrm{am}-6 \mathrm{am}^{\mathrm{c}}$ & 0.31 & 0.46 & 0.00 & 1.00 \\
\hline Weekend ${ }^{\mathrm{c}}$ & 0.26 & 0.44 & 0.00 & 1.00 \\
\hline Yearc $^{\mathrm{C}}$ & 2010.95 & 1.98 & 2008.00 & 2014.00 \\
\hline Prev. prescribed (\#) ${ }^{\mathrm{d}}$ & 3.49 & 9.22 & 0.00 & 133.00 \\
\hline \multicolumn{5}{|l|}{ Demographic Variables } \\
\hline Female & 0.57 & 0.50 & 0.00 & 1.00 \\
\hline Age & 44.81 & 16.85 & 19.02 & 100.00 \\
\hline \multicolumn{5}{|l|}{ Race } \\
\hline White & 0.29 & 0.45 & 0.00 & 1.00 \\
\hline Black & 0.60 & 0.49 & 0.00 & 1.00 \\
\hline Latino & 0.07 & 0.26 & 0.00 & 1.00 \\
\hline Asian & 0.01 & 0.10 & 0.00 & 1.00 \\
\hline Other & 0.03 & 0.18 & 0.00 & 1.00 \\
\hline \multicolumn{5}{|l|}{ Marital Status } \\
\hline Single & 0.53 & 0.50 & 0.00 & 1.00 \\
\hline Married & 0.27 & 0.44 & 0.00 & 1.00 \\
\hline Divorced & 0.09 & 0.29 & 0.00 & 1.00 \\
\hline Widowed & 0.07 & 0.26 & 0.00 & 1.00 \\
\hline Separated & 0.04 & 0.20 & 0.00 & 1.00 \\
\hline
\end{tabular}

Notes: Sample includes all EMR from hospital ED ( $\mathrm{n}=180,829$ events; 63,513 unique individuals). a) Whether visit resulted in an opioid prescription. b) Number of emergency department patients in last 4 hours. c) Time of visit. d) Number of opioids ever prescribed to patient during previous emergency department visits. 
Table 2 Logistic Regression Models for Opioid Prescription during Emergency Department Visit

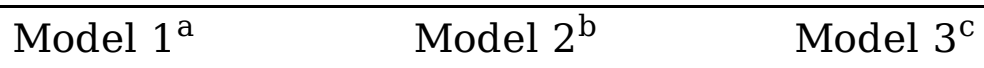

\section{Contextual Variables}

ED crowding ${ }^{\mathrm{d}}$

ED crowding $x$ Black

12am-6am

Weekend

Year

Prev. prescribed (\#)

Demographic Variables
$-0.002$

0.000

0.025

0.023

$-0.020$

0.050
$-0.001$

0.000

$0.012 * * *$

$0.014^{* * *}$

$-0.024 * * *$

0.037

$* * *$

0.023

0.028

$* * *$

$-0.034$

$* * *$

$-0.047$

Age 60+

Race $^{\mathrm{e}}$

\section{Black}

Latino

Asian

Other

Marital Status ${ }^{f}$

Married

Divorced

Widowed

Separated

Sex

Female

Female x Black

Female $x$ Latino

Female $x$ Asian

Female $x$ Other

Intercept

$\begin{array}{rlrl}-0.017 & * * & -0.005 & \\ 0.015 * & -0.009 & \\ -0.068 & * * * & -0.055 & * * * \\ 0.007 & & -0.003 & \end{array}$

$-0.002$

0.000

$0.020^{* * *}$

$0.021 * * *$

$-0.038 * * *$

$0.027^{* * *}$ 
average marginal effects. Full models include polynomial terms and interaction effects between prev. prescribed (\#) and year. Sample includes all electronic medical records from hospital's emergency department ( $\mathrm{n}=180,829$ events; 63,513 unique individuals). Years of analysis $=2008-2014$. a) Includes within-person random effects. b) Includes within-person random effects and ICD-9 diagnosis. c) Includes within-person fixed effects and ICD-9 diagnosis. d) Number of emergency department patients in last 4 hours. e) Reference $=$ White. f) Reference $=$ Unmarried.

\section{Figures}

Average Number ED Visits by Day of Year

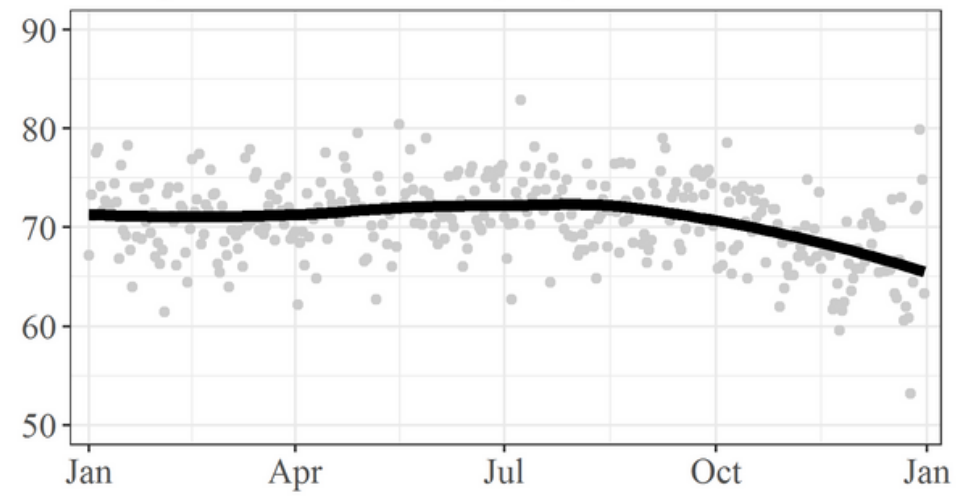

Average Number ED Visits by Day of Week

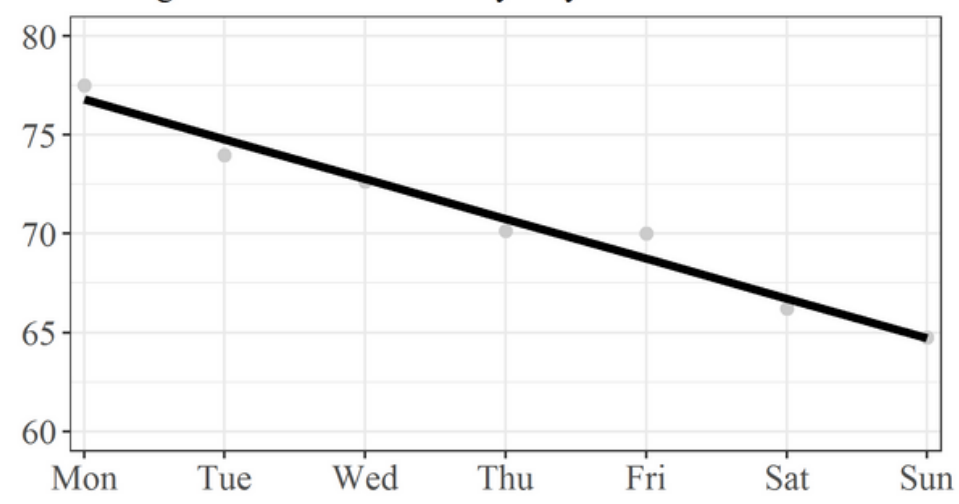

Average Number ED Visits by Day of Month

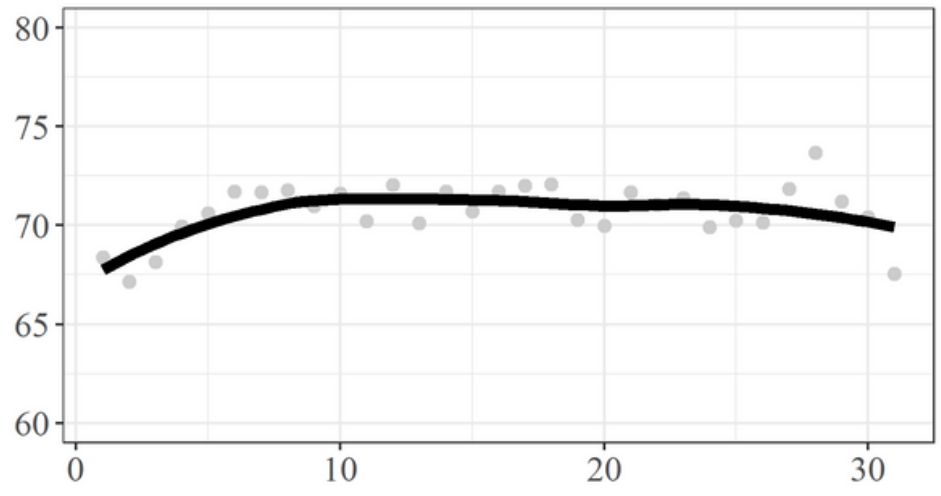

Average Number ED Visits by Hour of Day

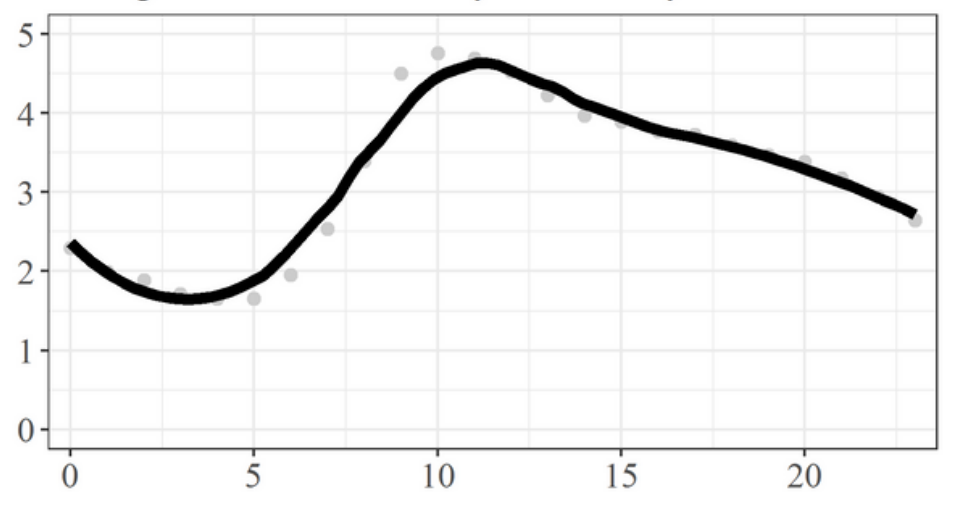

Figure 1

Number of Emergency Department (ED) Visits by Unit of Time Note: Sample includes all electronic medical records from hospital's emergency department ( $n=180,829$ events; 63,513 unique individuals). Points indicate individual estimates; lines indicate locally estimated scatterplot smoothing (LOESS) curves. 